\title{
Assessing predictors for the success of GnRH antagonist protocol in reproductive women in IVF/ICSI - in fresh cycles
}

\author{
AN-CONG WANG ${ }^{1,2}$, YING WANG ${ }^{3}$, FENG-XIA WU $^{4}$ and DONG-YI ZHU ${ }^{1,2}$ \\ Departments of ${ }^{1}$ Reproductive Medicine and ${ }^{2}$ Obstetrics and Gynecology, Linyi People's Hospital, Linyi, Shandong 276003; \\ ${ }^{3}$ Department of Gynecologic Oncology, Shandong Cancer Hospital and Institute, Jinan, Shandong 250117; \\ ${ }^{4}$ Department of Anatomy, Shandong University, Jinan, Shandong 250012, P.R. China
}

Received July 31, 2017; Accepted September 6, 2017

DOI: $10.3892 / \mathrm{br} .2017 .984$

\begin{abstract}
The aim of the present study was to evaluate the factors that affect the success rate of gonadotropin-releasing hormone antagonist on in vitro fertilization/intracytoplasmic sperm injection cycles. Multivariate analysis was performed to assess the factors that influence the outcomes, such as oocytes retrieved, and the success of pregnancy. The results showed that $\mathrm{E}_{2}, \mathrm{P}$ on human chorionic gonadotropin (HCG) day and body mass index (BMI) were positively correlated with the number of oocytes retrieved $(\mathrm{P}=0.001, \mathrm{P}=0.024, \mathrm{P}=0.017$, respectively). The duration of infertility as well as the luteinizing hormone on $\mathrm{HCG}$ day were negatively correlated with the number of oocytes $(\mathrm{P}=0.048, \mathrm{P}=0.002$, respectively). The age of the women and $\mathrm{P}$ on $\mathrm{HCG}$ day were negatively correlated with successful pregnancy $(\mathrm{P}<0.001, \mathrm{P}=0.022)$. In conclusion, some parameters, such as $\mathrm{E}_{2}, \mathrm{P}$, and $\mathrm{LH}$ on the HCG day, as well as age and BMI, may affect treatment outcomes.
\end{abstract}

\section{Introduction}

Gonadotropin-releasing hormone (GnRH) antagonist is used in controlled ovarian stimulation cycles, and is at least as safe as the long GnRH agonist protocol. Furthermore, the GnRH antagonist protocol requires less follicle stimulation, and has a lower risk for ovarian hyperstimulation syndrome (1). It has been shown that age, oocyte, quality of the embryo and endometrial receptivity are the most important factors in the success of in vitro fertilization (IVF), and the GnRH antagonist has an extrapituitary effect on the above factors (2).

The GnRH antagonist offers several advantages compared with the GnRH agonist protocol, including a reduction in the duration of the treatment and reduced doses of gonadotropins,

Correspondence to: Dr An-Cong Wang, Department of Reproductive Medicine, Linyi People's Hospital, 27 Jiefang Road, Linyi, Shandong 276003, P.R. China

E-mail: ancongw12@163.com

Key words: IVF/ICSI-ET, GnRH antagonist, clinical pregnancy, fresh cycle as well as no risk of ovarian cyst formation (3). Qiao et al reported that the normal responders treated with the GnRH antagonist protocol exhibited the same high success rates as women treated with the long GnRH agonist protocol (4). However, in low responders, the ovaries are stimulated without pituitary suppression, which may induce asynchronous follicular development. Antral follicle size during the early follicular phase is markedly heterogeneous. As GnRH antagonists prevent the increase in luteinizing hormone (LH) levels, it has been reported that the $\mathrm{GnRH}$ antagonist causes more profound LH than follicle-stimulating hormone (FSH) blockage, thereby reducing the follicular fluid $\mathrm{E}_{2}$ level compared to $\mathrm{GnRH}$ agonist protocols (5). Thus, whether the levels of $\mathrm{LH}$ and $\mathrm{E}_{2}$ on human chorionic gonadotropin (HCG) day influence the outcomes of the antagonist protocol need to be elucidated.

As FSH is needed for ensuring follicular growth as well as endometrial development and LH is needed for achieving proper oocyte maturation, both FSH and LH are necessary for oocyte development. However, the role of baseline serum LH in predicting the success of IVF in terms of infertility outcomes remains controversial. Inconsistent results have been found, especially with regard to infertile women aged $\geq 35$ years, regardless of whether they were using rLH supplementation during ovarian stimulation or not (6).

For the best results in IVF outcome, optimal follicular development should be achieved. It has been reported that good morphological quality of embryos is positively associated with conception after IVF (7) and endometrial characteristics, such as endometrial thickness (EM), have been evaluated as prognostic factors. However, the lack of consensus on EM is explained by the difficulty of an exact definition of a thin endometrium as assessed by transvaginal ultrasonography. Thus there is no clear conclusion on the clinical significance of EM as a predictive parameter for the chances of pregnancy after IVF.

Therefore, the aim of this retrospective study was to explore rFSH treatment without rLH supplementation and antagonist treatment and to evaluate the best predictors for the success of the GnRH antagonist protocol in reproductive women undergoing IVF/intracytoplasmic sperm injection (ICSI)-ET treatment in fresh cycles. Baseline serum FSH, LH, $\mathrm{E}_{2}$, P level and $\mathrm{LH}, \mathrm{E}_{2}$, P level and EM on the HCG day as well as the number of quality embryos, were evaluated to determine the 
influence of these factors on the success of IVF in terms of clinical pregnancy.

\section{Materials and methods}

Patients. This was a retrospective, single-centre cohort study. The study included patients who underwent IVF/ICSI cycles with the GnRH antagonist protocol from January 1, 2014 to September 30, 2015. All the patients reached the ovum pick-up stage. Only women undergoing their first antagonist protocol were included. It has been shown that the two sources (IVF/ICSI) of embryos have no effect on their cultivation (8). The fecundity of all male partners of the patients was normal according to World Health Organization criteria (9). The present study was conducted at the Linyi People's Hospital (Shandong, China) and was approved by the Ethics Committee of Linyi People's Hospital. A written informed consent form was obtained from all patients.

Materials. The patients underwent $\mathrm{COH}$ using the flexible GnRH antagonist protocol, as reported in previous studies $(10,11)$. The injection of $\mathrm{rFSH}\left(\right.$ Gonal $\mathrm{F}^{\circledR}$; Merck Serono S.A., Geneva, Switzerland) started on day 2 of the menstrual cycle, and the starting dose of rFSH was 75-300 IU daily and was individualized according to the patient's age, body mass index (BMI), antral follicle count, baseline $\mathrm{E}_{2}, \mathrm{P}, \mathrm{FSH}$ and $\mathrm{LH}$ concentration.

The GnRH antagonist used was cetrorelix acetate (Cetrotide; Merck-Serono Ltd., Aubonne, Switzerland). Treatment with rFSH and cetrorelix acetate was continued until the day of the final oocyte maturation trigger.

For the group, the final oocyte maturation trigger was injected with $250 \mu \mathrm{g}$ human chorionic gonadotropin $\alpha$ (r-HCG), (Ovidrel, Merck-Serono Ltd., Aubonne, Switzerland). Thirty-six to $37 \mathrm{~h}$ after $\mathrm{r}-\mathrm{HCG}$ injection, oocyte aspiration was performed, which was guided by transvaginal ultrasound. Embryo transfer was performed on day 3/5 following oocyte retrieval. All of the cycles received luteal phase support with progesterone in the form of vaginal suppositories of $90 \mathrm{mg}$ once daily (Crinone, Fleet Laboratories Ltd., Watford, UK). Clinical pregnancy was defined as visualization of a gestational sac and foetal cardiac activity on transvaginal ultrasound after 4-5 weeks of IVF-ET.

Statistical analysis. SPSS 23.0 software for Windows was used for statistical treatment (SPSS, Chicago, IL, USA). In the present study, the data were reported as the mean \pm standard deviation (SD). Linear regression was conducted to evaluate the effect of the different variables on the number of oocytes retrieved. Logistic regression was conducted to evaluate the different variables on the success of pregnancy. A $\mathrm{P}<0.05$ was considered significant.

\section{Results}

Baseline characteristics. A total of 298 IVF/ICSI fresh cycles were evaluated in antagonist protocols. The serum gonadotropin (FSH and $\mathrm{LH}$ ), $\mathrm{E}_{2}$ and $\mathrm{P}$ levels were evaluated beyond the baseline, and the baseline characteristics are presented in Table I. The mean age was 35.46 \pm 5.22 (108 cases
Table I. Baseline cycle characteristics of patients undergoing the $\mathrm{GnRH}$ antagonist protocol.

\begin{tabular}{lc}
\hline Characteristics & Mean \pm SD \\
\hline Age (years) & $35.46 \pm 5.22$ \\
Duration of infertility (years) & $4.25 \pm 3.09$ \\
BMI (kg/m²) & $24.03 \pm 3.22$ \\
Basal FSH level (UI/l) & $8.11 \pm 2.70$ \\
Basal LH level (UI/l) & $3.80 \pm 2.04$ \\
Basal E 2 (pg/ml) & $45.58 \pm 20.98$ \\
Basal P (ng/ml) & $0.48 \pm 0.28$ \\
Initial dose of rFSH administration (UI) & $204.82 \pm 47.61$ \\
Total dose of rFSH (UI) & $1926.22 \pm 589.11$ \\
Stimulation duration (days) & $9.29 \pm 1.71$ \\
Total dose of antagonist administration (mg) & $1.01 \pm 0.39$ \\
E on HCG day (pg/ml) & $1411.26 \pm 936.37$ \\
P on HCG day (ng/ml) & $0.62 \pm 0.29$ \\
LH on HCG day (UI/l) & $2.57 \pm 2.07$ \\
EM on HCG day (mm) & $9.98 \pm 2.20$ \\
Mean no. of total oocytes retrieved & $5.12 \pm 3.31$ \\
Mean no. of 2PN oocytes & $3.70 \pm 2.80$ \\
Mean no. of embryos available & $2.28 \pm 1.31$ \\
Mean no. of high quality embryo & $1.55 \pm 1.49$ \\
Mean no. of embryos transferred & $1.73 \pm 0.50$ \\
\hline
\end{tabular}

GnRH, gonadotropin-releasing hormone; BMI, body mass index; FSH, follicle-stimulating hormone; LH, luteinizing hormone; HCG, human chorionic gonadotropin; EM, endometrial thickness.

aged $<35$ years, 190 cases aged $\geq 35$ years), and the mean BMI was $24.03 \pm 3.22$. The mean duration of infertility was $4.25 \pm 3.09$ years, and the mean basal hormone profile of FSH was $8.11 \pm 2.70, \mathrm{E}_{2}$ was $45.58 \pm 20.98, \mathrm{P}$ was $0.48 \pm 0.28$ and the mean LH was $3.80 \pm 2.04$. The mean initial dose of rFSH administration was $204.82 \pm 47.61$, and the mean total dose of $\mathrm{rFSH}$ was $1926.22 \pm 589.11$. The mean duration of stimulation was 9.29 \pm 1.71 . The total dose of antagonist administered was $1.01 \pm 0.39$. The mean level of $\mathrm{E}_{2}$ on $\mathrm{HCG}$ day was 1411.26 \pm 936.37 , and the mean level of P on HCG day was $0.62 \pm 0.29$. The mean level of LH on HCG day was 2.57 \pm 2.07 , and the mean thickness of the EM on HCG day was 9.98 2.20. The mean number of total oocytes retrieved was $5.12 \pm 3.31$, and the mean number of $2 \mathrm{PN}$ oocytes was $3.70 \pm 2.80$. The mean number of embryos available was $2.28 \pm 1.31$, the mean number of high quality embryos was $1.55 \pm 1.49$, and the mean number of embryos transferred was $1.73 \pm 0.50$. The clinical pregnancy rate was $38.93 \%$ (116/298), and the live birth rate was $26.85 \%(80 / 298)$ (Table I).

Linear regression for the number of total oocytes retrieved and logistic regression analysis for the success of clinical pregnancy. Table II shows the influence factors that affect the number of oocytes retrieved. The duration of infertility and the level of LH on HCG day was negatively correlated with the number of oocytes retrieved $(\mathrm{P}=0.048, \mathrm{P}=0.002$, respectively). By contrast, the level of $\mathrm{E}_{2}, \mathrm{P}$ on $\mathrm{HCG}$ day and the BMI were 
Table II. Linear regression analysis for the number of total oocytes retrieved.

\begin{tabular}{lrc}
\hline Items & $\beta$ & P-value \\
\hline Age (years) & -0.070 & 0.087 \\
Duration of infertility (years) & -0.078 & $0.048^{\mathrm{a}}$ \\
BMI $\left(\mathrm{kg} / \mathrm{m}^{2}\right)$ & 0.102 & $0.017^{\mathrm{b}}$ \\
Basal FSH (UI/l) & -0.083 & 0.055 \\
Basal LH (UI/l) & 0.025 & 0.555 \\
Basal $\mathrm{E}_{2}(\mathrm{pg} / \mathrm{ml})$ & -0.034 & 0.396 \\
Basal P (ng/ml) & 0.005 & 0.888 \\
Initial dose of rFSH administration (UI) & 0.067 & 0.557 \\
Total dose of rFSH (UI) & -0.072 & 0.650 \\
Duration of rFSH stimulation (days) & -0.003 & 0.981 \\
Total dose of antagonist (mg) & 0.077 & 0.150 \\
E $_{2}$ on HCG day (pg/ml) & 0.667 & $<0.001^{\mathrm{b}}$ \\
P on HCG day (ng/ml) & 0.096 & $0.024^{\mathrm{b}}$ \\
LH on HCG day (UI/l) & -0.122 & $0.002^{\mathrm{a}}$ \\
EM on HCG day (cm) & 0.067 & 0.079 \\
\hline
\end{tabular}

${ }^{a}$ Negatively and ${ }^{b}$ Positively correlated with total oocytes retrieved. BMI, body mass index; FSH, follicle-stimulating hormone; LH, luteinizing hormone; HCG, human chorionic gonadotropin; EM, endometrial thickness.

positively correlated with the number of oocytes retrieved ( $\mathrm{P}<0.001, \mathrm{P}=0.024, \mathrm{P}=0.017$, respectively).

Table III shows that age was inversely correlated with the success of pregnancy $(\mathrm{P}<0.001)$, as well as the level of $\mathrm{P}$ on HCG day $(\mathrm{P}=0.022)$.

\section{Discussion}

In this retrospective study, we aimed to identify factors that affect the success of the antagonist protocol to improve clinical outcomes. Usually clinicians focus on factors such as the duration of $\mathrm{rFSH}$ and antagonist, $\mathrm{E}_{2} / \mathrm{P} / \mathrm{LH}$ levels on the HCG day, and EM on the HCG day when these protocols were used to treat patients.

In this study, we found that there were no significant correlated factors among the age of the women, basal $\mathrm{FSH} / \mathrm{LH} / \mathrm{E}_{2} / \mathrm{P}$ levels, EM on HCG day, or duration of $\mathrm{rFSH}$ administration on the number of total oocytes retrieved.

There was noteworthy finding that the level of $\mathrm{E}_{2}, \mathrm{P}$ on HCG day was positively correlated with the number of oocytes retrieved. Another finding in the study was the positive correlation between the BMI of the women and the number of oocytes retrieved. The duration of infertility was negatively correlated with the number of eggs retrieved.

It has been reported that clinical pregnancy rates increase with the increasing EM, and most studies have defined the minimal thickness at approximately $7 \mathrm{~mm}$. When women fail to achieve minimal endometrial development, the embryos are often frozen (12-14). However, in the retrospective study, the mean EM on HCG day was $9.98 \pm 2.20 \mathrm{~mm}$ and exhibited no significant correlation with the success of pregnancy.
Table III. Logistic regression analysis for the success of clinical pregnancy.

\begin{tabular}{lrrl}
\hline Items & \multicolumn{1}{c}{$\beta$} & Exp (B) & P-value \\
\hline Age (years) & -0.116 & 0.890 & $0.000^{\mathrm{a}}$ \\
Duration of infertility (years) & -0.020 & 0.980 & 0.660 \\
BMI (kg/m ${ }^{2}$ ) & -0.020 & 0.981 & 0.682 \\
Basal FSH (UI/l) & 0.046 & 1.047 & 0.423 \\
Basal LH (UI/l) & -0.068 & 0.934 & 0.341 \\
Basal E $(\mathrm{pg} / \mathrm{ml})$ & -0.008 & 0.992 & 0.265 \\
Basal P (ng/ml) & -0.012 & 0.988 & 0.980 \\
Initial dose of rFSH & -0.007 & 0.993 & 0.413 \\
administration (UI) & & & \\
Total dose of rFSH (UI) & 0.001 & 1.001 & 0.468 \\
Duration of rFSH & -0.308 & 0.735 & 0.231 \\
stimulation (days) & & & \\
Total dose of antagonist & 0.827 & 2.286 & 0.093 \\
administration (mg) & & & \\
E $_{2}$ on HCG day (pg/ml) & 0.000 & 1.000 & 0.594 \\
Pon HCG day (ng/ml) & -1.266 & 0.282 & $0.022^{\text {a }}$ \\
LH on HCG day (UI/l) & 0.033 & 1.034 & 0.603 \\
EM on HCG day (cm) & 0.020 & 1.021 & 0.742 \\
Mean no. of total oocytes retrieved & -0.080 & 0.923 & 0.391 \\
Mean no. of 2PN oocytes & 0.155 & 1.167 & 0.142 \\
Mean no. of embryos available & -0.206 & 0.814 & 0.417 \\
Mean no. of high quality embryo & 0.339 & 1.403 & 0.078 \\
Mean no. of embryos transferred & 0.507 & 1.660 & 0.114 \\
\hline
\end{tabular}

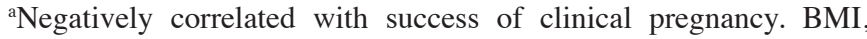
body mass index; FSH, follicle-stimulating hormone; LH, luteinizing hormone; HCG, human chorionic gonadotropin; EM, endometrial thickness.

In controlled ovarian stimulation, the use of pituitary suppression agents to prevent premature LH surge is central. GnRH antagonists permit the immediate suppression of pituitary gonadotrophins by competitive inhibition of gonadotrophin receptors. However, low endogenous LH concentrations may induce negative effects that affect treatment outcomes. It has been reported that low LH levels increase early pregnancy loss rates $(15,16)$, but the definition of the LH threshold remains controversial. Westergaard et al used an LH threshold of $0.5 \mathrm{mIU} / \mathrm{ml}$ to define the low LH group and found similar clinical pregnancy rates between the low LH group and the normal group (17). However, the low LH group was associated with a lower chance of live birth compared with the normal group. Chen et al used an LH cut-off value of $0.8 \mathrm{mIU} / \mathrm{ml}$ to define the two groups $(\leq 0.8 \mathrm{mIU} / \mathrm{ml}$ and $>0.8 \mathrm{mIU} / \mathrm{ml})(18)$. Their study showed significantly increased early pregnancy loss rates in the low LH group $(\leq 0.8 \mathrm{mIU} / \mathrm{ml})$ compared with the group with normal LH concentrations $(>0.8 \mathrm{mIU} / \mathrm{ml})$. Although the implantation rates, clinical pregnancy rates and live birth rates were lower in the low LH group, the differences were not significant.

In the present study, the mean basal LH was $3.80 \pm 2.04$, and the LH on HCG day was $2.57 \pm 2.07$. Linear regression showed 
that basal LH had no effect on the oocytes retrieved, but the LH on HCG day was inversely correlated with the number of eggs retrieved. Using logistic regression, neither the basal LH nor the LH on HCG day was significantly correlated with the success of clinical pregnancy.

In IVF/ICSI cycles, the endometrium and embryo are exposed to supraphysiological concentrations of $\mathrm{E}_{2}$ and $\mathrm{P}$, which may influence pregnancy outcomes. Whether the elevated $\mathrm{E}_{2}$, and especially the $\mathrm{P}$ level, affect the clinical pregnancy is still controversial. Li et al reported that the $\mathrm{P}$ concentration on HCG day adversely affects IVF pregnancy outcomes (19). However, some findings have shown that elevated P levels have no effect on blastocyst embryo transfer in GnRH agonist/antagonist cycles $(20,21)$. Some investigators have demonstrated that there is no correlation between $\mathrm{P}$ levels and pregnancy outcome (22-24). Yang et al demonstrated that the high P level on HCG day may affect the endometrial receptivity rather than the oocyte or embryo quality (25). For those patients, researchers have suggested frozen-thawed embryo transfer or blastocyst transfer. Li et al have demonstrated the effect of high progesterone levels on outcomes of in vitro fertilization-embryo transfer in patients with different ovarian responses (26). They defined a P cut-off value of $2.5 \mathrm{ng} / \mathrm{ml}$ in the high ovarian response group, $2.25 \mathrm{ng} / \mathrm{ml}$ in the moderate ovarian response group and $1.5 \mathrm{ng} / \mathrm{ml}$ in the low ovarian response group. Their results showed that in each group, the clinical pregnancy rate and embryo implantation rate were lower in the patients with an increased P level than those in which the $\mathrm{P}$ level did not increase. The increased level of $\mathrm{P}$ on the day of HCG may affect the treatment outcomes of IVF-ET. The phenomenon of the increased $\mathrm{P}$ level in women undergoing controlled ovarian stimulation still needs to be elucidated. It has been reported that for women with a low ovarian response and, a P level of $>1.5 \mathrm{ng} / \mathrm{ml}, \mathrm{HCG}$ given in advance reduces the negative effects of the elevated $\mathrm{P}$ on pregnancy outcomes. For PCOS patients with a high ovarian response, a small starting dose of FSH may reduce an elevated P level $(27,28)$.

In the present study, the mean serum $P$ level was $0.62 \pm 0.29$, and the P level on HCG day was inversely correlated with the success of clinical pregnancy.

As the serum $E_{2}$ level reflects the maturity of the follicle and the quality of the oocytes, the $\mathrm{E}_{2}$ level is regarded as one of the most important factors in predicting clinical outcomes during the course of IVF/ICSI cycles.

Peña et al reported that patients with $\mathrm{E}_{2}>3,000 \mathrm{pg} / \mathrm{ml}$ on HCG days had a significantly higher number of oocytes retrieved than other groups with lower $E_{2}$ levels of 1,500-3,000 pg/ml and $<1,500 \mathrm{pg} / \mathrm{ml}$ (29). Some researchers have concluded that $\mathrm{E}_{2}>4,000 \mathrm{pg} / \mathrm{ml}$ or $\mathrm{E}_{2}>5,000 \mathrm{pg} / \mathrm{ml}$ on the day of HCG had a significantly higher number of oocytes retrieved compared to other groups $(30,31)$. Siddhartha et al (32) conducted a retrospective study on the association of supraphysiological $E_{2}$ level with the reproductive outcome of ICSI. The study subjects were grouped based on the serum $\mathrm{E}_{2}$ level on the day of HCG: group 1, <1,000 pg/ml; group 2, 1,000-2,000 pg/ml; group 3, 2,000.1-3,000 pg/ml; group 4, 3,000.1-4,000 pg/ml; group 5, $4,000.1-5,000 \mathrm{pg} / \mathrm{ml}$. Their results showed that group $5 \mathrm{had}$ a significantly higher number of oocytes retrieved than groups 1 and 2 . However, there were no significant differences in the pregnancy rates.
In our retrospective study, the $\mathrm{E}_{2}$ on the day of $\mathrm{HCG}$ was positively correlated with oocytes retrieved, but the $\mathrm{E}_{2}$ on the day of HCG was not correlated with pregnancy rates and could not be used to predict pregnancy in antagonist cycles, which is consistent with previous findings (29-33).

In the present study, the mean age was $35.46 \pm 5.22$ and the mean number of oocytes retrieved was $5.12 \pm 3.31$. Our results indicated that $63.76 \%$ of women (190/298) undergoing antagonist protocols were aged $\geq 35$ years. This explains the results such as the low $\mathrm{E}_{2}$ level on HCG day (1411.26 \pm 936.37$)$ and low total oocytes retrieved $(5.12 \pm 3.31)$.

The finding suggests, that age was negatively correlated with the success of pregnancy, is consistent with that of other clinicians (34).

In conclusion, further larger studies should be performed to elucidate the role of factors such as the EM, $\mathrm{E}_{2}$ and $\mathrm{P}$ level on $\mathrm{HCG}$ day in predicting the number of embryos available and clinical outcomes. Additionally, we hope that an increased number of predictors may be identified to guide the use of $\mathrm{GnRH}$ antagonists to ensure good reproductive and clinical outcomes.

\section{Acknowledgements}

The authors thank the administrative staff in the Department of Reproductive Medicine in Linyi People's Hospital for their support. The study was supported by the National Natural Science Fund of China (grant no.81501620) and the Shandong Provincial Medical and Health Science and Technology Development Project, China (grant no. 2015WS0377).

\section{References}

1. Tarlatzis BC, Fauser BC, Kolibianakis EM, Diedrich K, Rombauts L and Devroey P: GnRH antagonists in ovarian stimulation for IVF. Hum Reprod Update 12: 333-340, 2006.

2. Hernandez ER: Embryo implantation and GnRH antagonists: embryo implantation: the Rubicon for GnRH antagonists. Hum Reprod 15: 1211-1216, 2000.

3. Devroey P, Aboulghar M, Garcia-Velasco J, Griesinger G, Humaidan P, Kolibianakis E, Ledger W, Tomás C and Fauser BC: Improving the patient's experience of IVF/ICSI: A proposal for an ovarian stimulation protocol with GnRH antagonist co-treatment. Hum Reprod 24: 764-774, 2009.

4. Qiao J, Lu G, Zhang HW, Chen H, Ma C, Olofsson JI, Witjes H, Heijnen E and Mannaerts B: A randomized controlled trial of the GnRH antagonist ganirelix in Chinese normal responders: High efficacy and pregnancy rates. Gynecol Endocrinol 28: 800-804, 2012.

5. Matikainen T, Ding YQ, Vergara M, Huhtaniemi I, Couzinet B and Schaison G: Differing responses of plasma bioactive and immunoreactive follicle-stimulating hormone and luteinizing hormone to gonadotropin-releasing hormone antagonist and agonist treatments in postmenopausal women. J Clin Endocrinol Metab 75: 820-825, 1992.

6. Hill MJ, Levens ED, Levy G, Ryan ME, Csokmay JM, DeCherney AH and Whitcomb BW: The use of recombinant luteinizing hormone in patients undergoing assisted reproductive techniques with advanced reproductive age: A systematic review and meta-analysis. Fertil Steril 97: 1108-14.e1, 2012.

7. Laasch $\mathrm{C}$ and Puscheck E: Cumulative embryo score, not endometrial thickness, is best for pregnancy prediction in IVF. J Assist Reprod Genet 21: 47-50, 2004.

8. Aytoz A, Van den Abbeel E, Bonduelle M, Camus M, Joris H, Van Steirteghem A and Devroey P: Obstetric outcome of pregnancies after the transfer of cryopreserved and fresh embryos obtained by conventional in-vitro fertilization and intracytoplasmic sperm injection. Hum Reprod 14: 2619-2624, 1999. 
9. Cooper TG, Noonan E, von Eckardstein S, Auger J, Baker HW, Behre HM, Haugen TB, Kruger T, Wang C, Mbizvo MT, et al World Health Organization reference values for human semen characteristics. Hum Reprod Update 16: 231-245, 2010.

10. Lee TH, Wu MY, Chen HF, Chen MJ, Ho HN and Yang YS: Ovarian response and follicular development for single-dose and multiple-dose protocols for gonadotropin-releasing hormone antagonist administration. Fertil Steril 83: 1700-1707, 2005.

11. Depalo R, Lorusso F, Palmisano M, Bassi E, Totaro I, Vacca M, Trerotoli P, Masciandaro P and Selvaggi L: Follicular growth and oocyte maturation in GnRH agonist and antagonist protocols for in vitro fertilisation and embryo transfer. Gynecol Endocrinol 25: 328-334, 2009.

12. Kovacs P, Matyas S, Boda K and Kaali SG: The effect of endometrial thickness on IVF/ICSI outcome. Hum Reprod 18 : 2337-2341, 2003.

13. Bozdag G, Esinler I and Yarali H: The impact of endometrial thickness and texture on intracytoplasmic sperm injection outcome. J Reprod Med 54: 303-311, 2009

14. Abdalla HI, Brooks AA, Johnson MR, Kirkland A, Thomas A and Studd JW: Endometrial thickness: A predictor of implantation in ovum recipients? Hum Reprod 9: 363-365, 1994.

15. Tesarik J, Hazout A and Mendoza C: Luteinizing hormone affects uterine receptivity independently of ovarian function. Reprod Biomed Online 7: 59-64, 2003.

16. Zamah AM, Hsieh M, Chen J, Vigne JL, Rosen MP, Cedars M and Conti M: Human oocyte maturation is dependent on LH-stimulated accumulation of the epidermal growth factor-like growth factor, amphiregulin. Hum Reprod 25: 2569-2578, 2010.

17. Westergaard LG, Laursen SB and Andersen CY: Increased risk of early pregnancy loss by profound suppression of luteinizing hormone during ovarian stimulation in normogonadotrophic women undergoing assisted reproduction. Hum Reprod 15: 1003-1008, 2000.

18. Chen CD, Chiang YT, Yang PK, Chen MJ, Chang CH, Yang YS and Chen SU: Frequency of low serum LH is associated with increased early pregnancy loss in IVF/ICSI cycles. Reprod Biomed Online 33: 449-457, 2016.

19. Li R, Qiao J, Wang L, Zhen X and Lu Y: Serum progesterone concentration on day of HCG administration and IVF outcome. Reprod Biomed Online 16: 627-631, 2008.

20. Elgindy EA: Progesterone level and progesterone/estradiol ratio on the day of hCG administration: Detrimental cutoff levels and new treatment strategy. Fertil Steril 95: 1639-1644, 2011.

21. Orvieto R, Nahum R, Meltzer S, Liberty G, Anteby EY and Zohav E: GnRH agonist versus GnRH antagonist in ovarian stimulation: The role of elevated peak serum progesterone levels. Gynecol Endocrinol 29: 843-845, 2013.

22. Xu B, Li Z, Zhang H, Jin L, Li Y, Ai J and Zhu G: Serum progesterone level effects on the outcome of in vitro fertilization in patients with different ovarian response: An analysis of more than 10,000 cycles. Fertil Steril 97: 1321-7.e1, 4, 2012.
23. Yding Andersen C, Bungum L, Nyboe Andersen A and Humaidan P: Preovulatory progesterone concentration associates significantly to follicle number and $\mathrm{LH}$ concentration but not to pregnancy rate. Reprod Biomed Online 23: 187-195, 2011.

24. Moffitt DV, Queenan JT Jr, Shaw R and Muasher SJ: Progesterone levels on the day of human chorionic gonadotropin do not predict pregnancy outcome from the transfer of fresh or cryopreserved embryos from the same cohort. Fertil Steril 67: 296-301, 1997.

25. Yang S, Pang T, Li R, Yang R, Zhen X, Chen X, Wang H, Ma C, Liu P and Qiao J: The individualized choice of embryo transfer timing for patients with elevated serum progesterone level on the HCG day in IVF/ICSI cycles: A prospective randomized clinical study. Gynecol Endocrinol 31: 355-358, 2015.

26. Li PF, Zhu H, Tan L, Zhao DM, Ma LY, Xiang YG, Zhang D, Dou Q and Lu N: Effects of high progesterone on outcomes of in vitro fertilization-embryo transfer in patients with different ovarian responses. Syst Biol Reprod Med 61: 161-167, 2015.

27. Harada T, Katagiri C, Takao N, Toda T, Mio Y and Terakawa N: Altering the timing of human chorionic gonadotropin injection according to serum progesterone $(\mathrm{P})$ concentrations improves embryo quality in cycles with subtle P rise. Fertil Steril 65: 594-597, 1996.

28. Filicori M, Cognigni GE, Gamberini E, Parmegiani L, Troilo E and Roset B: Efficacy of low-dose human chorionic gonadotropin alone to complete controlled ovarian stimulation. Fertil Steril 84: 394-401, 2005.

29. Peña JE, Chang PL, Chan LK, Zeitoun K, Thornton MH II and Sauer MV: Supraphysiological estradiol levels do not affect oocyte and embryo quality in oocyte donation cycles. Hum Reprod 17: 83-87, 2002.

30. Wu CH, Kuo TC, Wu HH, Yeh GP and Tsai HD: High serum estradiol levels are not detrimental to in vitro fertilization outcome. Taiwan J Obstet Gynecol 46: 54-59, 2007.

31. Kara M, Kutlu T, Sofuoglu K, Devranoglu B and Cetinkaya T: Association between serum estradiol level on the hCG administration day and IVF-ICSI outcome. Iran J Reprod Med 10: 53-58, 2012.

32. Siddhartha N, Reddy NS, Pandurangi M, Tamizharasi M, Radha V and Kanimozhi K: Correlation of serum estradiol level on the day of ovulation trigger with the reproductive outcome of intracytoplasmic sperm injection. J Hum Reprod Sci 9: 23-27, 2016.

33. Taşkin EA, Atabekoğlu CS, Musali N, Öztuna D and Sönmezer M: Association of serum estradiol levels on the day of hCG administration with pregnancy rates and embryo scores in fresh ICSI/ET cycles down regulated with either GnRH agonists or GnRH antagonists. Arch Gynecol Obstet 289: 399-405, 2014.

34. Griffiths A, Dyer SM, Lord SJ, Pardy C, Fraser IS and Eckermann S: A cost-effectiveness analysis of in-vitro fertilization by maternal age and number of treatment attempts. Hum Reprod 25: 924-931, 2010. 